\title{
The effect of growth interruption on the properties of InGaAs/InAIAs quantum well structures
}

\author{
W. C. H. Choy, P. J. Hughes, and B. L. Weiss \\ School of Electronic Engineering, Information Technology and Mathematics, University of Surrey, \\ Guildford, Surrey GU2 5XH, United Kingdom \\ E. H. Li \\ Department of Electrical and Electronic Engineering, University of Hong Kong, Pokfulam Road, \\ Hong Kong \\ K. Hong and D. Pavlidis ${ }^{\text {a) }}$ \\ Department of Electrical Engineering and Computer Science, University of Michigan, Ann Arbor, \\ Michigan 48109-2122
}

(Received 1 July 1997; accepted for publication 14 November 1997)

\begin{abstract}
The effect of the growth interruption time during the growth of InGaAs/InAlAs quantum well structures is shown to have a significant effect on both the interband transitions, as determined by photoreflectance, and the electrical properties of the as-grown structure. The results show that, for increasing growth interruption time, the quantum well heterointerfaces become more abrupt and the carrier mobility increases, thereby demonstrating that long interruption times are preferable for the growth of high quality rectangular quantum well structures. (C) 1998 American Institute of Physics. [S0003-6951(98)01403-X]
\end{abstract}

The growth of quantum well $(\mathrm{QW})$ heterostructures is critical as it determines the shape of the QW interfaces and therefore their optical and electronic properties. It is generally assumed that the profile of as-grown QWs is rectangular, although studies ${ }^{1,2}$ have found evidence for asymmetry in the QW shape which has resulted in significant changes in the properties of such QW structures.

In this work, the effect of growth interruption times (GITs) on the optical and electrical properties of an InGaAs/ InAlAs QW structure is investigated; as differences in GITs are expected to play an important role in determining the shape of the QW profile. Electrical transport properties of these structures as a function of GITs were determined from Hall measurements. Room temperature photoreflectance (PR) was used here to observe the behaviour of QW interband transition energies, which are sensitive to the QW profile, with different GITs. This technique is a powerful post growth characterization tool to analyze the subband structure of QWs. ${ }^{3,4}$ A semi-empirical model of subband structure is developed here to identify the transition energies of the InGaAs/InAlAs QW structure.

Three identical InGaAs/InAlAs QW structure with different GITs were grown by MOVPE. Trimethylindium, trimethylgallium, and trimethylaluminum were used for In, Ga, and Al sources, respectively, and $100 \%$ arsine and phosphine was used for group $\mathrm{V}$ elements. Each structure consisted of a $60 \mathrm{~nm} \quad \mathrm{In}_{0.507} \mathrm{Al}_{0.493} \mathrm{As}$ upper barrier layer, a $25 \mathrm{~nm}$ $\mathrm{In}_{0.53} \mathrm{Ga}_{0.47} \mathrm{As}$ well layer, and a $300 \mathrm{~nm} \mathrm{In}_{0.507} \mathrm{Al}_{0.493} \mathrm{As}$ lower barrier layer grown on an SI InP substrate. All investigated structures reported in this work were undoped. The GITs used here were $0 \mathrm{~s}, 5 \mathrm{~s}$, and $15 \mathrm{~s}$ when switching from barrier growth to well growth and vice versa. All layers were grown at $650{ }^{\circ} \mathrm{C}$, which was found to be the optimum temperature for low background carrier concentration.

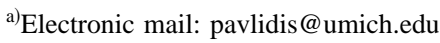

A one-dimensional, one-particle, Schrodinger-like wave equation obtained from an envelope function approximation, ${ }^{5}$ using a Ben-Denial and Duke model $^{6}$ is solved numerically to determine the subband of this structure. The band-gap energy and carrier effective masses are fitted from Ref. 7 and 8 respectively, see Table I. The band offset ratio is taken as 72:28. ${ }^{9}$ Other parameters are fitted by Vegard's law linearly using the parameters of binary compounds including AlAs, InAs, and GaAs.

The PR system has been reported previously. ${ }^{10}$ The PR spectra of the as-grown QW samples as a function of GITs are shown in Fig. 1. Also shown in Fig. 1 are the calculated transition energies from the model assuming a rectangular QW profile. To simplify the picture only the electron $(\mathrm{m})$ to heavy-hole $(\mathrm{n}),\left(\mathrm{H}_{m n}\right)$, transitions are indicated. The InP substrate and InAlAs barrier signatures at $\sim 1.48 \mathrm{eV}$ and $\sim 1.347 \mathrm{eV}$, respectively, are readily identified and serve to calibrate the spectra. From Fig. 1, the higher order transitions appear to shift higher in energy with increasing GITs. The

TABLE I. Material parameters used in the modeling calculations. $E_{g}$ is the band-gap energy, $m_{e}$ and $m_{\mathrm{hh}}$ are the effective masses of electron and heavy hole, respectively.

\begin{tabular}{|c|c|c|}
\hline & Unit & $\mathrm{In}_{x} \mathrm{Al}_{y} \mathrm{Ga}_{1-x-y} \mathrm{As}$ \\
\hline$E_{g}$ & $\mathrm{eV}$ & $\begin{array}{c}0.354 x+3.017 y+1.432(1-x-y)-0.99 x y \\
-0.51 x(1-x-y)+0.04 y(1-x-y)\end{array}$ \\
\hline$m_{e}$ & $m_{0}$ & $\begin{array}{l}\left(x y m_{\mathrm{eInAlAs}}+y(1-x-y) m_{\mathrm{eAIGa} A \mathrm{As}}\right. \\
\left.\quad+x(1-x-y) m_{\mathrm{eInGaAs}}\right) /\left(x+y-x y-x^{2}-y^{2}\right) \\
\quad m_{\mathrm{eInAlAs}}=0.1719-0.1506((1+x-y) / 2) \\
m_{\mathrm{eAIGa} A \mathrm{As}}=0.0632+0.0856(x / 2+y)+0.0231(x / 2+y)^{2} \\
m_{\mathrm{eInGaAs}}=0.0632-0.0419(x+y / 2)\end{array}$ \\
\hline$m_{\mathrm{hh}}$ & $m_{0}$ & $\begin{array}{l}\left(x y m_{\mathrm{hh} \mathrm{InAlAs}}+y(1-x-y) m_{\mathrm{hh} \mathrm{AlGa} A \mathrm{As}}\right. \\
\left.\quad+x(1-x-y) m_{\mathrm{hh} \mathrm{InGaAs}}\right) /\left(x+y-x y-x^{2}-y^{2}\right) \\
\quad m_{\mathrm{hh} \mathrm{InAlAs}}=0.145-0.121((1+x-y) / 2) \\
\quad m_{\mathrm{hh} \mathrm{AlGa} A \mathrm{As}}=0.50+0.2(x / 2+y) \\
\quad m_{\mathrm{hh} \mathrm{InGaAs}}=0.5-0.09(x+y / 2)\end{array}$ \\
\hline
\end{tabular}




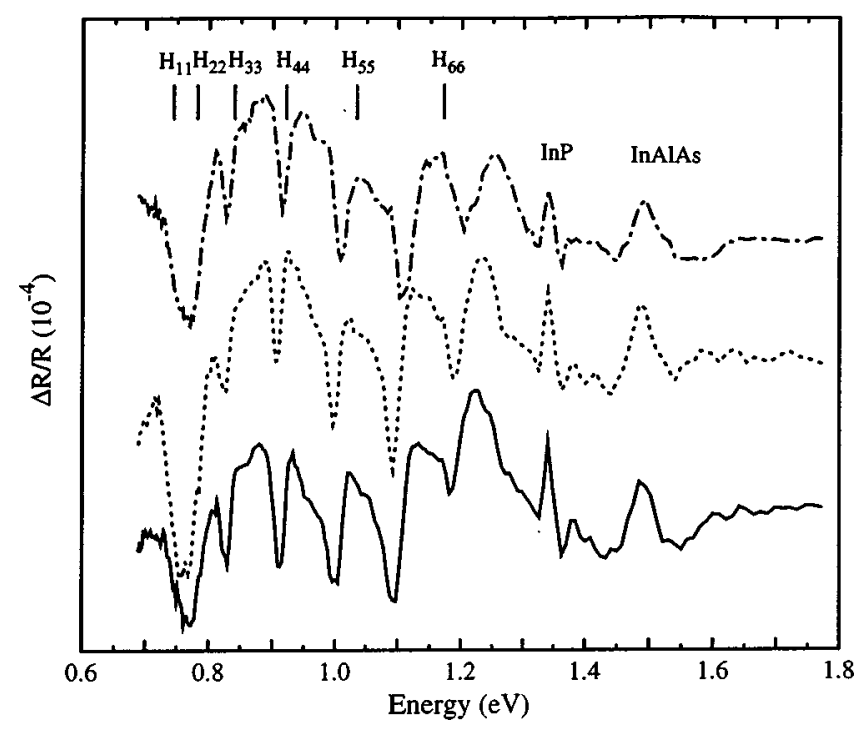

FIG. 1. The PR spectra for the three as-grown QW structures for GIT values of $0 \mathrm{~s}(-), 5 \mathrm{~s}(\ldots \ldots \ldots)$, and $15 \mathrm{~s}(\cdots \cdots)$. The energies determined for the electron $(m)$ to heavy-hole $(n) \mathrm{H}_{m n}$ transitions are indicated.

increase of the higher order transitions with GIT indicates that a significant change in the shape of the QW profile has occurred. The degree to which this profile changes can be inferred by modeling two profiles; rectangular (RQW) and exponential (EQW). The PR energy shifts suggests that QW profile becomes more abrupt, i.e., rectangular, with increasing GIT. Consequently, the structure with GITs of 15 and 0 s were modeled by RQW and EQW profiles, respectively, as shown in Fig. 3. The EQW profile is symmetric and is defined, with central point $=0$, as follows:

$$
x=x_{0} \exp \left(\frac{\beta}{z^{2}-\left(\frac{1}{2} L_{z}^{\text {bottom }}\right)^{2}}\right)+C, \quad|z| \geqslant 0,
$$

where $L_{z}^{\text {bottom }}, z, x_{0}$ and $C$ are the well width at the bottom, the growth direction, the Al concentration in barrier and constant parameter respectively. The EQW profile is defined by the curvature parameter $\beta$, so that small values of $\beta$ correspond to steep profiles. From Fig. 3, the change in QW profile to produce energy shifts comparable to those deduced

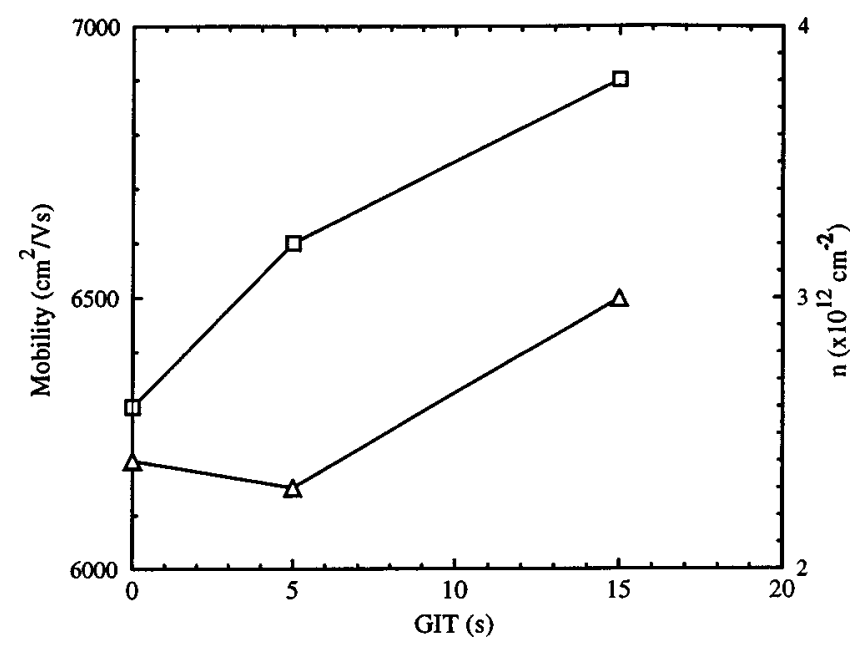

FIG. 2. The Hall mobility $(\square)$ and corresponding background carrier density $(\triangle)$ as a function of GITs.
TABLE II. Calculated transition energies of the RQW and EQW profiles.

\begin{tabular}{ccc}
\hline \hline Transition & $\begin{array}{c}\text { RQW } \\
(\mathrm{eV})\end{array}$ & $\begin{array}{c}\text { EQW } \\
(\mathrm{eV})\end{array}$ \\
\hline $\mathrm{H}_{11}$ & 0.745 & 0.744 \\
$\mathrm{H}_{22}$ & 0.781 & 0.779 \\
$\mathrm{H}_{33}$ & 0.842 & 0.837 \\
$\mathrm{H}_{44}$ & 0.927 & 0.918 \\
$\mathrm{H}_{55}$ & 1.038 & 1.022 \\
$\mathrm{H}_{66}$ & 1.171 & 1.147 \\
\hline \hline
\end{tabular}

from PR ( $\leqslant 20 \mathrm{meV}$ ), occurs primarily at the top of the QW. The corresponding transition energies calculated from the model for both profiles are given in Table II.

The Hall mobility and background carrier density as a function of GIT are shown in Fig. 2. The data presented in this figure correspond to the two-dimensional electron gas formed in the quantum well as a result of nonintentionallydoped (NID) InAlAs, InGaAs. These plots show that both the mobility and the sheet carrier concentration increase with GIT. The increase is attributed to the removal of defects which scatter carriers and causes a decrease in their mobility. In addition, a large GIT allows the material to grow and order itself as near to a single crystal as possible.

In summary, these results show that increasing the growth interruption time from 0 to $15 \mathrm{~s}$ increases the abruptness of the well barrier interfaces and results in an increase of the carrier mobility of the corresponding two-dimensional electron gas. The resulting change in shape at the top of the QW leaves the ground state transition energies unchanged, while the higher order transition energies are modified. This, in effect, results in a change of the optical properties while maintaining the same band-gap of the QW structure. In addition, the PR results show how this technique can be used simply as an effective characterization tool to evaluate the quality of the QW heterointerfaces as determined by the QW transitions.

While rectangular QWs are desirable for the in depth understanding of the material and devices, nonideal structures with graded interfaces may in fact be preferable for devices as the less abrupt interfaces enables fast carrier removal for high speed switching.

This project is supported in part by HKU-CRCG ARPA COST (MDA 972-94-1-0004) and URI (DAAL 03-92-G-

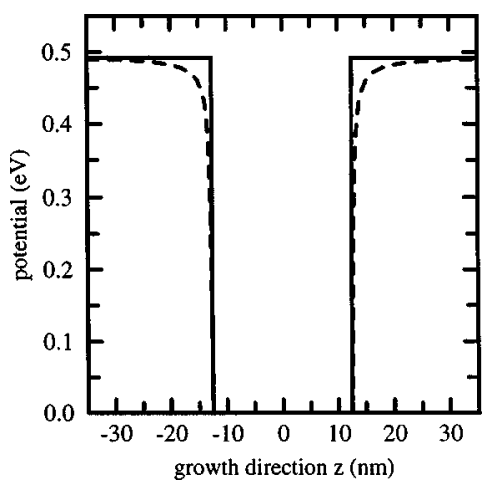

FIG. 3. The conduction subband structure of RQW (- - ) and EQW (-----) profiles. A $\beta$ value of 5 was used to model the EQW profile. 
0109). One of the authors (W. C. H. Choy) acknowledges the financial support of the Croucher Foundation.

${ }^{1}$ M. A. Herman, D. Bimberg, and J. Christen, J. Appl. Phys. 70, R1 (1991).

${ }^{2}$ P. M. Young and H. Ehrenreich, Appl. Phys. Lett. 61, 1069 (1992).

${ }^{3}$ H. Qiang, F. H. Pollak, Y.-S. Huang, W. S. Chi, R. Droopad, D. L. Mathine, and G. N. Maracas, Proc. SPIE 2139, 11 (1995).

${ }^{4}$ P. J. Hughes, T. J. C. Hosea, and B. L. Weiss, Semicond. Sci. Technol. 10, 1339 (1995).
${ }^{5}$ G. Bastard and J. A. Brum, IEEE J. Quantum Electron. 22, 113 (1987).

${ }^{6}$ D. J. BenDaniel and C. B. Duke, Phys. Rev. 152, 683 (1966).

${ }^{7}$ T. Ishikawa and J. E. Bowers. IEEE J. Quantum Electron. 30, 562 (1994).

${ }^{8}$ H. Nagai, S. Adachi, and T. Fukui, III-V Group Mixed Crystals, (Corona, Tokyo, 1990).

${ }^{9}$ S. Monéger, Y. Baltagi, T. Benyattou, A. Tabata, B. Ragot, G. Guillot, A. Georgakilas, K. Zekentes, and G. Halkias, J. Appl. Phys. 74, 1437 (1993).

${ }^{10}$ P. J. Hughes, B. L. Weiss, and T. J. C. Hosea, J. Appl. Phys. 77, 6472 (1995). 